\title{
Specific and Non-specific Defense against Parasitic Attack
}

\author{
Steven A. Frank* \\ Department of Ecology and Evolutionary Biology, University of California, Irvine, \\ CA 92697-2525, U.S.A.
}

(Received on 29 April 1999, Accepted in revised form on 11 November 1999)

\begin{abstract}
Specific defense protects against some parasite genotypes but not others, whereas non-specific defense is effective against all genotypes of a parasite. Some empirical studies observe hosts with variability only in non-specific defense, other studies find only specific defense. I analyse a model with combined specific and non-specific defense to determine the conditions that favor detectable variation in each form of defense. High variation in non-specific defense is often maintained when resistance increases in an accelerating way with investment, whereas low variation tends to occur when resistance increases at a decelerating rate with investment. Variation in specific defense rises as the parasite pays a higher cost to attack a broad host range (high cost of virulence), as the number of alternative specificities declines, and as the average level of non-specific defense increases. The last condition occurs because greater non-specific protection tends to stabilize the gene frequency dynamics of specific defense. Selection favors a negative association between costly components of specific and non-specific defense-hosts defended by one component are favored if they have reduced allocation to other costly components. A negative association confounds the measurement of costs of resistance. Individuals with specific defense may have reduced investment in costly nonspecific defense. This leads to an apparent advantage of specifically defended hosts in the absence of parasites and a measured cost of resistance that is negative.
\end{abstract}

(C) 2000 Academic Press

\section{Introduction}

Specific defense occurs when a host repels attack by some parasitic genotypes but not by others. Specificity may, for example, be mediated by a biochemical match between host receptors and parasite surface molecules. The match induces the host's non-specific defenses near the site of attack. This approach localizes the induced response and minimizes wasted defensive effort.

Non-specific defense protects against all parasitic genotypes of an attacking species. Such broad-band defense is sometimes called "durable

\footnotetext{
*E-mail:safrank@uci.edu
}

resistance" because it remains effective over time, whereas specific defense often succumbs to novel parasitic genotypes that evade recognition. Durable resistance has the obvious advantages of broad and continuous defense but may sometimes require constitutive and costly expression of defensive systems.

Many models of specific and non-specific resistance have studied evolutionary dynamics and maintenance of polymorphism. Models typically focus on either specific or non-specific resistance; here I examine the interaction between these two forms of defense. It seems likely that there would be some interaction-for example, highly successful specific and localized defense may 
influence the level of constitutive expression. However, interactions between epidemiology and genetics have sometimes proved to be counterintuitive.

\section{Biological Examples of Specific and Non-specific Defense}

Agricultural studies of plant-pathogen interactions provide the best data on specific and non-specific defense. Specificity is defined by statistical description of variation in attack and defense for a given sample of hosts and parasites (Vanderplank, 1963, 1984). Specific resistance is the variation in host fitness explained by an interaction between host and parasite genotype. The remaining variation in host response to parasitic attack is independent of parasite race - this is non-specific resistance.

Resistance in plant-pathogen interactions is often manifest by a hypersensitive response - the accumulation of defensive compounds in the tissue surrounding the point of invasion and an associated confinement of pathogen proliferation (Gabriel \& Rolfe, 1990). Successful pathogens, differing by a single mutation from resistant interactions (Kearney et al., 1988), do not elicit the hypersensitive response.

Biochemical models suggest that resistance occurs only when a pathogen allele produces a particular gene product (elicitor) that can be recognized by a matching host receptor (Gabriel \& Rolfe, 1990). If an elicitor-receptor match occurs, then the host induces a non-specific hypersensitive response and resists attack. If the same pathogen elicitor is present, but the host produces a non-matching receptor, then disease develops. Infection also succeeds when a pathogen lacks an elicitor that matches the specific host receptor.

The mechanisms of durable, non-specific resistance are less clear. Pathogens may contain a monomorphic elicitor that is essential for function and cannot be changed. Hosts may have a low level of constitutive expression of the nonspecific defensive system that provides partial resistance against all pathogen races. Or the hosts may lower the threshold signal at which a hypersensitive response is induced, leading to universal resistance against attack but possibly frequent "false alarms" in which defense is triggered in response to a harmless invasion or signal. Finally, mechanical or other biochemical mechanisms may be continuously expressed in a non-specific way. Most non-specific mechanisms suggest costly expression, although some may be relatively free of deleterious side effects.

The main point here is that mixed specific and non-specific defensive systems occur. The plant-pathogen interaction is a particularly wellstudied case, but there are many other examples of induced defense in response to signals of invasion or danger (Harvell, 1990; Karban \& Baldwin, 1997; Tollrian \& Harvell, 1999). When these signals of attack are recognized in a specific way, then resistance may be composed of specific recognition followed by induction of nonspecific defense. Such a two-part resistance is likely to be associated with widespread variation in the relative contributions of specific and nonspecific defense toward explaining the observed distribution of resistance.

In plant-pathogen systems, specific variation is commonly observed in both agricultural and natural populations (reviewed by Burdon, 1987; Frank, 1992, 1997). Non-specific defense is widely sought after and sometimes observed in agricultural systems (Vanderplank, 1984). The classic example is the interaction between potato blight and potato. The two potato varieties Maritta and Kennebec have the same profile of specific and complete resistance against several pathogen races but differ uniformly (non-specifically) in the severity of damage caused by successful infection. Such non-specific resistance would, however, have to be reclassified as specific if a newly analysed pathogen race overcame what had previously been classified as non-specific defense. Thus, classification depends partly on the intensity and duration of sampling.

Many studies have analysed variation in hosts' resistance to different parasite isolates and the host range of those parasite isolates over the sampled hosts. Patterns and generalities remain obscure. I illustrate this literature by two recent examples.

Fellowes et al. (1998) used laboratory selection experiments to study the resistance of Drosophila melanogaster to the endoparasitoid Leptopilina boulardi. They selected four replicate populations 
of the host for increased resistance to the parasitoid. Resistance increased from $0.5 \%$ to between 40 and $50 \%$ in five generations. Hosts from the selected lines suffered lower larval survival in comparison with unselected competitors.

Insects resist attack by encapsulating the parasitoid with defensive cells (Salt, 1970). Resistance has two important steps: recognition of a foreign object as non-self and induction of the encapsulation process. It is not clear at present whether the increased resistance observed by Fellowes et al. (1998) resulted from improved recognition or a more vigorous induced response. Genetic variation in insect resistance against endoparasitoids has been observed in several cases (Kraaijeveld et al., 1998), whereas there are only a few reports of variation in parasitoid success (summarized by Sasaki \& Godfray, 1999). If this pattern holds, variation in insect resistance would appear to be controlled by changes in the threshold and vigor of induced defense rather than by specific defense. Firm conclusions are premature, however, because inferring specific variation often requires intensive sampling.

Fellowes et al. (1999) expanded their study of D. melanogaster resistance. They selected different host lines for increased resistance to either of the endoparasitoids Asobara tabida or Leptopilina boulardi. They then tested each selected line against three parasitoids: the two from the selection experiments plus Leptopilina heterotoma. Lines selected against $L$. boulardi had increased resistance against all three parasitoid species. Lines selected against $A$. tabida had increased resistance against all parasitoids except L. boulardi. Apart from this last, unexplained exception, it appears that increased non-specific defense causes the enhanced protection of the selected lines.

Webster \& Woolhouse (1998) performed similar selection experiments on a snail and its schistosome parasites. They found nearly all the variation explained by enhanced specific defense. They conducted their study with the snail Biomphalaria glabrata, which is polymorphic in natural populations for resistance to Schistosoma mansoni. They selected two strains of the snail for increased or decreased resistance to two strains of the schistosome. Third-generation snails were tested for resistance against both parasite strains.
Each snail strain selected for higher or lower resistance to a particular parasite strain responded accordingly to that strain, but had no change in resistance to the other parasite strain. Thus variation in defense appears to be entirely specific. There was no trade-off in resistance, that is, increased resistance to one parasite strain had no association with resistance to the other parasite strain.

I draw four conclusions from the limited data available. First, protection against internal parasites often depends on recognition of invasion followed by induction of defense. Either phase, recognition or induced defense, may be specific or non-specific for a given sample of hosts and parasites.

Second, one cannot infer recognition or nonspecific defense when expressed in a constant way by all individuals of a given sample, even if such components of defense are critical aspects of a multistep process of resistance. Thus observed resistance always means variable resistance. The question is: Which factors favor variable specific defense and which factors favor variable nonspecific defense?

Third, the biochemical and physiological mechanisms of recognition and induced defense likely influence variability in resistance. For example, if successful recognition of an invading parasite can be turned to failure by small biochemical changes in elicitors and receptors, then specific recognition is likely to be highly variable. By contrast, if recognition depends on constant biochemical features of the parasites, then defense will appear as non-specific.

Fourth, given that much of the variation across host-parasite systems may depend on the presently unknown biochemistry and physiology of the interaction, the role of general models is necessarily limited. General models can clarify the broad categories of specific and non-specific defense that may occur, the conditions that maintain variable and thus observable resistance, and the potential interactions between different components of a defensive cascade.

\section{Model}

I use a system of Lotka-Volterra equations to model the host-parasite dynamics (e.g. May, 
1974). This allows joint study of fluctuating abundances caused by ecological dynamics (epidemiology) and fluctuating gene frequencies caused by host-parasite selection. I use a form that also includes competition among hosts for limited resources, imposing a carrying capacity on the hosts in the absence of parasites and avoiding the peculiar, neutrally stable limit cycles that occur in the absence of host competition.

The system is given by

$$
\begin{gathered}
\Delta h_{i j}=h_{i j}\left(r c_{i j}-r \sum_{x} \sum_{y} c_{x y} h_{x y} / K-m \sum_{k} \delta_{i j k} p_{k}\right) \Delta t \\
\Delta p_{k}=p_{k}\left(-s+b_{k} \sum_{i} \sum_{j} \delta_{i j k} h_{i j}\right) \Delta t
\end{gathered}
$$

The abundance of hosts with genotype $i j$ is given by $h_{i j}$. The host genotypes have varying aspects of specific and non-specific defense. For specific defense, each host carries a specific recognition allele, $i=0,1, \ldots, n$. For non-specific defense, each host carries an allele $j=0,1 / N, \ldots, N / N$, where the level of defense varies from 0 to 1 . A host that carries no defenses $(i=j=0)$ has an intrinsic rate of increase of $r$. Defenses have costs

$$
c_{i j}= \begin{cases}1-\alpha j, & i=0 \\ (1-a)(1-\alpha j), & i>0 .\end{cases}
$$

which are composed of two parts. Non-specific defense reduces the rate of increase by $1-\alpha j$, where $j$ is the level of constitutive expression and $\alpha$ is a cost parameter. Specific defense reduces the rate of increase by $1-a$ where $a$ is a cost parameter. The allele $i=0$ provides no specific defense and has no cost.

The middle term of eqn (1a) describes host competition-the pressure on a host's rate of increase caused by other hosts' tendency to increase, scaled by the carrying capacity of hosts, $K$.

The final term is the morbidity and mortality of parasitic attack, $m$, weighted by the success of attack over all parasites. Each parasite genotype $k$ succeeds against host genotype $i j$ according to

$$
\delta_{i j k}= \begin{cases}0, & i=k \neq 0 \\ 1-j^{z} & \text { otherwise }\end{cases}
$$

where specific resistance prevents attack when host and parasite have matching specificities, $i=k$, and the host is not carrying the null allele, $i=0$. When specific resistance fails, hosts resist according to their constitutive level of non-specific defense, $j^{z}$, where $z<1$ causes diminishing benefits with investment in constitutive defense, and $z>1$ causes accelerating benefits.

The time-scale of the interaction is set by the discrete time step $\Delta t$. As $\Delta t \rightarrow 0$, the interaction becomes continuous (differential) in time.

The abundance of parasites with genotype $k=0,1, \ldots, n$ is given by $p_{k}$. The death rate of parasites in the absence of hosts is $s$. The growth of parasites on successfully infected hosts is $b_{k}$, where $b_{k}=b$ for $k>0$ and $b_{0}=(1-v) b$. The zero allele has cost of virulence, $v$, and gains the advantage of universal host range because there is no matching host type that can provide specific resistance against this parasite genotype.

Non-dimensional analysis simplifies the system by reducing the number of parameters and clarifying the scaling relations among processes (Segel, 1972; Murray, 1989). I use the following substitutions:

$$
\begin{gathered}
\tau=r t, \quad \hat{h}=h / K, \quad \hat{p}=(m / r) p, \\
\hat{s}=s / r, \quad \hat{b}=b K / r,
\end{gathered}
$$

where the new variables are all non-dimensional quantities. The new time-scaling, $\tau$, expresses time in terms of the host population's potential rate of increase. For example, in a continuoustime model, the doubling time of the host population in the absence of competition or parasitism is $\ln (2) \approx 0.7$ non-dimensional time units. This transformed time-scaling holds for any set of parameters and provides easy comparison among systems that have different natural timescales.

The host abundances, $\hat{h}$, are fractions of the carrying capacity, which range from 0 to 1 . Again, this allows comparison on a common 
scale for models that assume different spatial distributions of resources and host competition. When I apply the substitution for parasite abundance, $\hat{p}$, below, the parameter $m$ disappears from the system. From this we can immediately deduce that morbidity and mortality per infection has no influence on ecological or evolutionary dynamics, but rather a lower value of $m$ simply translates into a proportionately higher abundance of parasites, that is, parasite pressure remains constant.

The final interesting point concerns the scaling for parasite growth rate. On the non-dimensional scale, $\hat{b}$ depends on the host's carrying capacity, $K$, showing the formal equivalence of increased parasite growth and increased host density on the increase of the parasite population.

I make these non-dimensional substitutions into eqns (1), expressing the system only in nondimensional quantities. For convenience, the hats are dropped from the non-dimensional parameters, and from this point all parameters are assumed to be non-dimensional quantities unless otherwise noted. The substitutions yield

$$
\begin{gathered}
\Delta h_{i j}=h_{i j}\left(c_{i j}-\sum_{x} \sum_{y} c_{x y} h_{x y}-\sum_{k} \delta_{i j k} p_{k}\right) \Delta \tau \\
\Delta p_{k}=p_{k}\left(-s+b_{k} \sum_{i} \sum_{j} \delta_{i j k} h_{i j}\right) \Delta \tau
\end{gathered}
$$

where the parameters $r, K$, and $m$ have been absorbed by the scaling relations.

\section{Special Cases}

To understand the interaction between nonspecific and specific defense one must first have an understanding of each process acting alone. The general model in eqns (4) subsumes four special cases.

\section{NON-SPECIFIC RESISTANCE}

Assume that hosts can defend non-specifically but lack the matching specificities of hostparasite recognition. In eqns (4) the matching specificities of host and parasite are set to $i=k=0$; in effect, the universal host-range allele of the parasite, $k=0$, has no cost, $v=0$, and thus goes to fixation. Hosts can vary at the non-specific locus indexed by $j=0,1 / N$, $\ldots, N / N$, thus $j$ varies between 0 and 1 and measures the fraction of maximal investment in defense.

The dynamics of non-specific defense are described by

$$
\begin{aligned}
& \Delta h_{j}=h_{j}\left[(1-\alpha j)-\sum_{k}(1-\alpha k) h_{k}-p\left(1-j^{z}\right)\right] \Delta \tau, \\
& \Delta p=p\left[-s+b \sum_{j}\left(1-j^{z}\right) h_{j}\right] \Delta \tau .
\end{aligned}
$$

Let $H$ be the total abundance of hosts. Then $h_{j} / H$ is the frequency of the $j$-th allele and $\sum_{j}\left(h_{j} / H\right) j=\bar{j}$ is the average level of expression in the population. Similarly, $\sum_{j}\left(h_{j} / H\right) j^{z}=\bar{j}^{z}$ is the average level of benefit provided by non-specific resistance.

With these definitions, the system can be written as

$$
\begin{gathered}
\Delta h_{j}=h_{j}\left[(1-\alpha j)-H(1-\alpha \bar{j})-p\left(1-j^{z}\right)\right] \Delta \tau \\
\Delta p=p\left[-s+b H\left(1-\bar{j}^{z}\right)\right] \Delta \tau
\end{gathered}
$$

and the equilibrium total abundance of hosts and parasites is given by

$$
\begin{gathered}
H^{*}=\frac{s}{b\left(1-\bar{j}^{z}\right)}, \\
P^{*}=\frac{(1-\alpha \bar{j})\left[b\left(1-\bar{j}^{z}\right)-s\right]}{b\left(1-\bar{j}^{z}\right)^{2}} .
\end{gathered}
$$

The distribution of genotypes must be analysed in two separate cases, for diminishing benefits with investment in non-specific resistance, $z<1$, and for accelerating benefits, $z>1$ [see Boots \& Haraguchi (1999) for a related analysis].

I first examine the case $z<1$ at equilibrium. Note that the dynamical equations for the different host genotypes have the form $\Delta h_{j}=h_{j} w_{j} \Delta \tau$, where $w_{j}$ is the rate of increase (fitness) of each type. Suppose that, for a given distribution of 
genotypes, we study the fitness of a single individual in a large population, considering how its fitness changes as a function of its genotype, $j$. The change in fitness with a small change in genotype is given by the derivative

$$
\frac{\mathrm{d} w_{j}}{\mathrm{~d} j}=-\alpha+z j^{z-1} p
$$

With $z<1$, the second derivative is negative, thus there may exist a single genotype, $0<j<1$, that has maximum fitness and is the monomorphic equilibrium. This candidate winner is found by solving $\mathrm{d} w_{j} / \mathrm{d} j=0$ at $j=j^{*}$ yielding the equation

$$
z j^{z-1}(1-\alpha j)\left[\left(1-j^{z}\right)-s / b\right]-\alpha\left(1-j^{z}\right)^{2}=0 .
$$

This must be solved numerically given the parameters $z, \alpha$, and $s / b$. I made a brief exploration of the parameter space and found that the equilibrium level of investment in non-specific defense, $j^{*}$, follows simple trends with respect to the parameters. In particular, as $s / b$ declines, $j^{*}$, increases-greater parasitic pressure implies a higher level of defense; as $\alpha$ increases, $j^{*}$ declines - greater cost of defense lowers the level of defense; and as $z$ increases, $j^{*}$ increases - as the marginal gains for defense decline more slowly with investment, the allocation to defense rises.

Interesting problems arise when considering how to measure and define resistance. One choice is to measure the fitness of an attacked individual divided by the fitness that individual would have if not attacked. If attack has no effect, then resistance is one; if attack kills the host, then resistance is zero. Although this measure appears reasonable at first glance, it is rarely used. Consider, for example, a population in which hosts always have their fitness reduced by one-half from attack. One would rarely describe the hosts as having 50\% resistance in this case. Many parasites damage but do not kill their hosts, and this interaction by itself is not considered an expression of resistance.

Variation in resistance is often a more interesting quantity. For example, a population responds to selection for increased resistance in proportion to its variance in resistance. Thus, a selection experiment that found a response mainly of non-specific defense suggests greater variability in non-specific than in specific defense for the sample of hosts and parasites studied. By contrast, individuals may invest heavily in costly non-specific defense but lack variation in that investment. In this case, different populations exposed to various levels of parasite pressure may have different levels of constant expression to non-specific defense.

The important point is that both the average and the variability of investment in non-specific defense are important quantities. The variation is relatively easy to measure within populations, whereas the average investment can only be inferred by comparison among populations.

The condition in eqn (6) describes the forces acting on average investment, $j^{*}$. What about variability? Numerical studies with mutation show that the stabilizing force of diminishing returns, with $z<1$, typically maintains a coefficient of variation about the equilibrium of less than $5 \%$. In addition, non-specific defense reduces parasite growth, which in turn reduces the tendency for non-equilibrium fluctuations in abundance. This ecological stability helps to maintain low levels of genetic variability in resistance.

The second case concerns accelerating benefits of resistance per unit investment, $z>1$. The second derivative of fitness with respect to phenotype, $\mathrm{d}^{2} w_{j} / \mathrm{d} j^{2}=z(z-1) j^{z-2} p$, is positive in this case implying disruptive selection. Thus, candidate equilibria include mixtures of minimum and maximum expression, $j=0$ and 1, respectively, or fixation of either type. Non-specific resistance appears as a qualitative factor even though there is a potential for quantitative variation.

The dynamics of the two host types and the parasite are given by

$$
\begin{gathered}
\Delta h_{0}=h_{0}\left[1-\left(h_{0}+(1-\alpha) h_{1}\right)-p\right] \Delta \tau, \\
\Delta h_{1}=h_{1}\left[1-\alpha-\left(h_{0}+(1-\alpha) h_{1}\right)\right] \Delta \tau, \\
\Delta p=p\left[-s+b h_{0}\right] \Delta \tau .
\end{gathered}
$$


The equilibrium is a mixture of minimum and maximum expression when the cost of nonspecific resistance is bounded such that $\alpha<1-s / b$, in which case the equilibrium is given by

$$
\begin{aligned}
& h_{0}^{*}=s / b, \\
& h_{1}^{*}=\frac{1-\alpha-s / b}{1-\alpha}, \\
& p^{*}=\alpha,
\end{aligned}
$$

with frequency of resistance, $h_{1}^{*} /\left(h_{0}^{*}+h_{1}^{*}\right)$, as

$$
f^{*}=\frac{1-\alpha-s / b}{1-\alpha-\alpha(s / b)}
$$

The equilibrium abundances present an interesting pattern. The abundance of the susceptible host, $h_{0}$, is independent of cost, $\alpha$. By contrast, the parasite, which can only attack the suceptible host, has equilibrium abundance equal to $\alpha$. The diagram in Fig. 1 shows how density-dependent competition among hosts causes this pattern of equilibrium abundances.

High parasite growth rates, $b$, and long time lags in interaction, $\Delta \tau$, destabilize the ecological interactions and cause boom and bust cycles of parasite epidemics. These epidemics tend to maintain high levels of resistance because an outbreak drives the frequency of resistance near fixation. The parasites may die off as resistance rises, followed by a decline in the frequency of resistance. This decline in resistance sets up another round of parasite boom and bust.

In summary, diminishing returns on defensive investment lead to limited quantitative polymorphisms about an intermediate level of expression. By contrast, accelerating benefits favor a polymorphism of high and low expression (Boots \& Haraguchi, 1999). Conditions favoring slow growth of parasites (low $b$ ) maintain a high frequency of non-expressing hosts with a low frequency of resistant hosts, whereas high host density and fast parasite growth (high $b$ ) favor a high frequency of resistance.

In the Discussion, I will consider these new results for non-specific resistance in light of the experiments by Fellowes et al. (1998, 1999). The

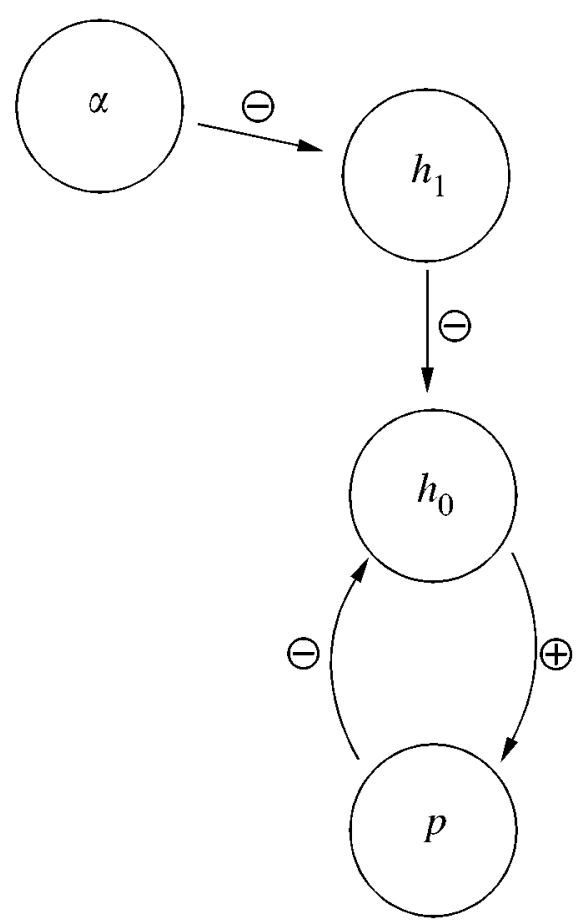

FIG. 1. The various effects caused by cost of non-specific resistance, $\alpha$. An increase in $\alpha$ directly reduces $h_{1}$. The effect of increasing $\alpha$ on parasite abundance, $p$, is obtained by multiplying the effects through the pathway: negative on $h_{1}$, negative on $h_{0}$ by density-dependent competition among hosts, and positive on $p$, yielding a net positive effect. Interestingly, increasing $\alpha$ has no net effect on the abundance of the susceptible host, $h_{0}$. This lack of effect arises from two opposing forces: the effect on $h_{0}$ is positive via reduction in competition with $h_{1}$ (the product of two negative paths from $\alpha$ to $h_{0}$ ), and the effect on $h_{0}$ is negative via an increase in parasite abundance, $p$.

following three sections review earlier models for specific defense. This review is useful because it brings together in a common framework different assumptions and methods of study. This background is also required to understand the interactions between specific and non-specific defense, which I describe after summarizing the models of specific defense.

\section{SPECIFIC DEFENSE: HAPLOID GENE-FOR-GENE}

Flor's (1971) classical gene-for-gene model states that for each locus in the host there is a matching locus in the parasite that conditions success of attack. The typical patterns of dominance and phenotypic interaction at each conditioning locus yield a $2 \times 2$ matrix such that a host has either a susceptible or a resistant phenotype 
and the pathogen has either a virulent or an avirulent phenotype. A resistant reaction for this gene pair only occurs with a match between the host resistance phenotype and the parasite arivulence phenotype. Any of the other three phenotypic combinations allows parasitic attack.

This pairwise gene-for-gene interaction occurs over all the matching gene pairs of the host and parasite. If the interaction leads to host resistance at any single matching gene pair, the net effect is resistance. A parasite succeeds only when all its avirulence phenotypes are matched by host susceptibility phenotypes. Note that plant pathologists use the word "virulence" to mean host range, whereas in other fields "virulence" means the aggressiveness of the parasite.

My model is haploid and there is only a single matching locus between host and parasite. We obtain the simplest form of a gene-for-gene matching specificity from eqns (4) by turning off non-specific defense, $j=0$, and limiting the number of matching specificities to $n=1$. Dropping the $j$ subscript for hosts, the full system is

$$
\begin{aligned}
\Delta h_{0}= & h_{0}\left[1-\left[h_{0}+(1-a) h_{1}\right]\right. \\
& \left.-\left(p_{0}+p_{1}\right)\right] \Delta \tau, \\
\Delta h_{1}= & h_{1}\left[1-a-\left[h_{0}+(1-a) h_{1}\right]-p_{0}\right] \Delta \tau, \\
\Delta p_{0}= & p_{0}\left[-s+b(1-v)\left(h_{0}+h_{1}\right)\right] \Delta \tau, \\
\Delta p_{1}= & p_{1}\left[-s+b h_{0}\right] \Delta \tau .
\end{aligned}
$$

The two top equations describe host susceptibility $\left(h_{0}\right)$ and resistance $\left(h_{1}\right)$; the two bottom equations describe parasite virulence $\left(p_{0}\right)$ and arivulence $\left(p_{1}\right)$. From earlier definitions, the cost of resistance is $a$; the cost of virulence is $v$, where virulence is more aptly called "universal host range". This is a special case of the multilocus model in Frank (1993a), according to which at equilibrium the relative abundances of resistance $(R)$ to susceptibility $(S)$ and virulence $(V)$ to avirulence $(A)$ are

$$
\begin{aligned}
& R: S=v: 1-v, \\
& V: A=1-a-H^{*}(1-a v): a,
\end{aligned}
$$

where $H^{*}=s /[b(1-v)]$ is the total equilibrium abundance of hosts. Note that there is a minor error in eqn (6) of Frank (1993a), in which $(1-a)^{N}\left(1-H^{*}\right)$ should be $(1-a)^{N}-$ $H^{*}(1-a v)^{N}$. This correction applies also to eqn (12) of Frank (1993a) and eqn (5) of Frank (1993b). This change does not affect in any way the conclusions from those earlier papers.

These equations emphasize the three key processes that influence the equilibrium frequencies of resistance and virulence. First, the frequency of host resistance rises with the cost of virulence, $v$, and is independent of the cost of resistance. Second, the frequency of virulence declines with the cost of resistance, $a$, which can be seen by rewriting the $V: A$ ratio as $1-a\left(1-v H^{*}\right)-H^{*}: a$ and noting that $1-v H^{*}>0$ when all types are present. Third, the cost of virulence influences the frequency of virulence only through the effect of density-dependent competition among hosts, which can be seen by rewriting the $V: A$ ratio as $1-a-\left[h_{0}^{*}+(1-a) h_{1}^{*}\right]: a$ and noting that the term $\left[h_{0}^{*}+(1-a) h_{1}^{*}\right]$ derives from host competition in the solution of $p_{0}^{*}$ from eqn ( $\left.8 \mathrm{~b}\right)$. In the absence of host competition, the $V: A$ ratio is $1-a: a$. Figure 2 provides an intuitive explanation for these effects. (Many other gene-for-gene models have been published. See the references in Leonard \& Czochor, 1980; Burdon, 1987; Frank, 1992, 1993a).

\section{SPECIFIC DEFENSE: MATCHING ALLELE}

The classical gene-for-gene model requires costs of resistance and virulence to maintain polymorphism. Without a cost of virulence, the universal host-range allele $(\mathrm{V})$ of the parasite goes to fixation; without a cost of resistance, the hosts' resistance allele $(\mathrm{R})$ goes to fixation. Alternatively, there may be a series of matching allelic specificities at each locus, which allows polymorphism to be maintained by frequency dependence.

I developed a formal model of matching specificities, which I called the matching-allele model (Frank, 1993b). The model is obtained from eqns (4) by assuming no non-specific defense and an absence of the special 0 alleles of the matching specificities such that the matching alleles vary as $i, k=1, \ldots, n$. These modifications are equivalent 


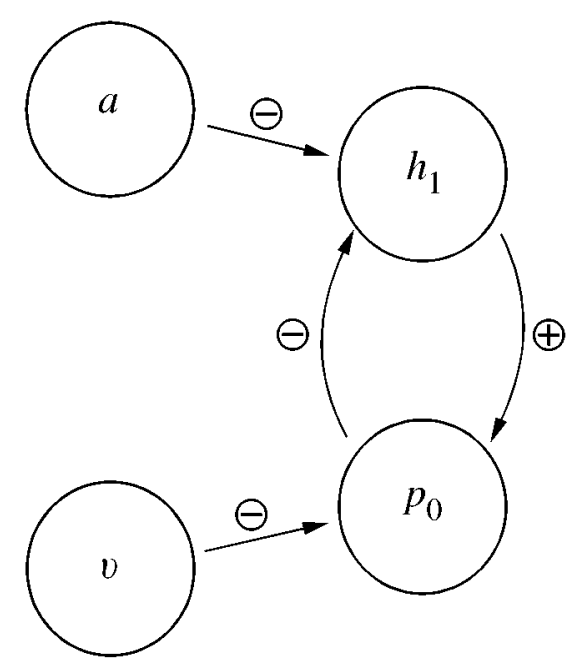

FIG. 2. The feedbacks in a gene-for-gene model of specific defense. A rise in the cost of resistance, $a$, directly lowers the abundance of resistant hosts, $h_{1}$, but indirectly raises $h_{1}$ through a feedback effect. The feedback is calculated by multiplying the individual effects of the three steps in the feedback loop: $a$ negatively influences, $h_{1}$, which in turn positively influences the virulent phenotype of the parasite, $p_{0}$, which negatively influences $h_{1}$. The net effect of a rise in $a$ is a decrease in $p_{0}$ and no change in $h_{1}$. Similar calculations show that an increase in $v$ raises $h_{1}$ but has no net effect on $p_{0}$ in this diagram. The effect of host competition is not shown here. The only effect of more intense host competition is to reduce the abundance of the virulent parasite phenotype because, by weakening the hosts, the parasites' ability to maintain a costly phenotype is reduced. This occurs because the parasites effectively graze excess host energy. Diagram modified from Frank (1992).

to assuming a very large cost of non-specific defense, $\alpha \rightarrow \infty$, no cost for host resistance alleles, $a=0$, and zero fitness for the parasite allele labeled as 0 that has universal host range, that is, the cost of virulence is $v=1$. With these assumptions, we have the system

$$
\begin{aligned}
& \Delta h_{i}=h_{i}\left[1-H-\left(P-p_{i}\right)\right] \Delta \tau, \\
& \Delta p_{k}=p_{k}\left[-s+b\left(H-h_{k}\right)\right] \Delta \tau,
\end{aligned}
$$

where $H$ and $P$ are the total abundances of hosts and parasites, and the genotypes range over $i, k=1, \ldots, n$. Frank (1993b) provided a full analysis. I summarize a few details as background for the extended model studied below.

The dynamics of the system are controlled by the equilibrium with all hosts and parasites present, which occurs at $h^{*}=s /[b(n-1)]$ and
$p^{*}=\left(1-H^{*}\right) /(n-1)$, where $H^{*}=n h^{*}$ and, by the symmetry of the system, $h_{i}^{*}=h^{*}$ and $p_{k}^{*}=p^{*}$ for all $i$ and $k$. This equilibrium point is unstable when there are discrete time lags $(\Delta \tau>0)$ in the competitive effects among hosts and in the interactions between host and parasite. This equilibrium is neutrally stable when interactions occur in continuous time $(\Delta \tau \rightarrow 0)$.

The most important aspect of dynamics concerns a qualitative change from limit cycles to colonization-extinction dynamics as the number of specificities, $n$, increases (Frank, 1993b, 1997). Systems with low $n$ maintain all specificities within the local population and follow nonlinear fluctuations governed by eqns (9). As the number of specificities rises, the average abundance per type declines. Lower average abundance increases the probability that one or more types become extinct locally.

Such extinctions make local populations prone to invasion and temporary dominance by colonists. For example, suppose that a particular parasite specificity was lost. Then, the matching host type will be driven to local extinction because it does not resist any parasite with which it interacts and has lower fitness than hosts resisting some of the locally present parasites. Loss of this host type sets up an opportunity for the matching parasite to invade by colonization; this parasite type will increase rapidly because no local hosts resist it. The colonizing parasite favors the reintroduction of the matching host type. The net effect is boom and bust cycles for matching types, with common local extinctions followed eventually by recolonizations.

\section{SPECIFIC DEFENSE: HYBRID MODEL}

Perhaps the most realistic model of specificity contains a mixture of gene-for-gene and matching-allele components (Frank, 1993b). On the gene-for-gene side, novel mutations to universal host range (virulence) are sometimes deletions (Flor, 1971), suggesting that virulence is the loss of a specific elicitor. Such deletions likely have a cost relative to various forms of the allele that act as elicitors, otherwise the deletions would quickly go to fixation. On the matching-allele side, it seems likely that small biochemical changes can alter a pathogen elicitor from a state 
that induces host defense to a state that avoids recognition and produces a successful infection. Small changes in the pathogen allele could be countered by matching host alleles, leading to a series of matching specificities.

A hybrid model with costly universal virulence and a series of cost-free matching specificities has not been studied. Within the present framework, such a model has the following form:

$$
\begin{aligned}
\Delta h_{0} & =h_{0}\left[1-\left((1-a) H+a h_{0}\right)-P\right] \Delta \tau, \\
\Delta h_{i} & =\mathrm{h}_{i}\left[1-a-\left((1-a) H+a h_{0}\right)-\left(P-p_{i}\right)\right] \Delta \tau, \\
\Delta p_{0} & =p_{0}[-s+b(1-v) H] \Delta \tau, \\
\Delta p_{k} & =p_{k}\left[-s+b\left(H-h_{k}\right)\right] \Delta \tau,
\end{aligned}
$$

where $H$ and $P$ are the total abundances of hosts and parasites, respectively. The host has a 0 allele that matches none of the parasites, the parasite has a 0 allele that escapes recognition by all hosts, and the matching specificities $i, k=1, \ldots, n$ cause resistance when $i=k$. The hosts' matching specificities have a cost $a$ relative to the null allele; the parasites' virulence allele has a cost $v$ relative to the matching specificities.

A candidate equilibrium with all types present occurs at

$$
\begin{aligned}
h_{0}^{*} & =\frac{s}{b(1-v)}(1-n v), \\
h_{i}^{*} & =\frac{s v}{b(1-v)}, \\
H^{*} & =\frac{s}{b(1-v)}, \\
p_{0}^{*} & =P^{*}-n a, \\
p_{i}^{*} & =a, \\
P^{*} & =1-\left[(1-a) H^{*}+a h_{0}^{*}\right],
\end{aligned}
$$

where this equilibrium exists when $v<1 / n$ and $a<P^{*} / n<1 / n$ and $P^{*}>0$. For the last condition, $b(1-v)>s$ is sufficient and converges to the necessary condition as $n$ increases. A full analysis of this model would be useful, but my focus here is on the interaction between specific and non-specific defense.

\section{Interaction between Specific and Non-specific Defense}

I focus on the three problems. First, what parameters favor qualitative polymorphism in non-specific defense and little polymorphism for specific defense? This matches the data of Fellowes et al. $(1998,1999)$ in which hosts succeed or fail qualitatively and non-specifically against various parasites.

Second, what parameters favor polymorphism of specific defense but little variation in nonspecific defense. This matches the data of Webster \& Woolhouse (1998) in which hosts were polymorphic for highly specific defense to different parasite genotypes but apparently lacked variation in non-specific defense.

Third, how does the interaction between specific and non-specific defense influence the measurement of the costs of resistance? The role of costs and the measurement of costs have been widely debated (Simms, 1992). Analysis of this model clarifies some of the difficulties involved.

To address these questions, I first introduce my methods of analysis. I then analyse the interactions between non-specific defense and the various forms of specific defense outlined in the previous section.

\section{PARAMETERS AND VARIABLES}

The model requires computer analysis. I describe the parameters, variables, and additional assumptions in this section.

The non-dimensional parameters in eqns (4) were described earlier; I summarize them here. The epidemiology is controlled by three parameters: $s$ and $b$ are the non-dimensionally scaled parasites' death and birth rates, and $\Delta \tau$ is the time step over which interactions occur. Recall that the non-dimensional parameter $b$ increases linearly with host density (carrying capacity), $K$, as described in eqn (3).

The cost of non-specific defense is $\alpha$. The benefit of non-specific defense is a power function of 
the amount of investment, $j^{z}$, where $j$ is investment and $z$ is an exponent that determines the benefit. The different levels of non-specific defense are coded by alternative alleles at a single locus. I use $N=127$, with non-specific defense varying over $j=0,1 / N, \ldots, N / N$.

The number of matching host and parasite specificities is $n$. Both host and parasite have an additional, null allele. For the host, this null allele matches none of the parasites (universal susceptibility). For the parasite, the null allele is matched by none of the hosts (universal host range). The hosts' cost of specific defense (for alleles other than the null allele) is $a$. The parasites' cost for the null allele that confers universal host range (virulence) is $v$.

The host has two haploid loci, one for specific defense and the other for non-specific defense. There are $n+1$ alleles for specific resistance and $N+1$ alleles for non-specific resistance, yielding $(N+1)(n+1)$ possible genotypes. The parasites have $n+1$ alternative alleles for specificity at their single locus. Thus, the system in eqns (4) has $(N+1)(n+1)^{2}$ equations. For all analyses I iterated these equations over discrete time steps of size $\Delta \tau$.

I assumed that any genotype that had an abundance below $10^{-8}$ was locally extinct. Locally extinct genotypes could be introduced by either immigration or mutation. For immigration, after each non-dimensional time unit had passed ( $1 / \Delta \tau$ iterations), I chose a random number on the interval $[0,1]$. If that number was less than the immigration rate, then a random host genotype was introduced with abundance $10^{-4}$. I then chose a second random number and, if it was less than the immigration rate, introduced a random parasite genotype. I fixed the immigration rate at $10^{-1}$ for all runs.

I also performed stepwise mutation on the hosts' quantitative trait for non-specific resistance. Each trait allele with value $j$ mutated to the next higher trait value, a step of $1 / N$, with probability $\mu / 2$, and to the next lower trait value with the same probability. For all runs, I set the mutation rate to $10^{-5}$ per non-dimensional time unit and performed mutation every $1 / \Delta \tau$ iterates.

In some runs I allowed recombination between the two host loci. Recombination was accomp- lished in the standard way by random mating between all haploid genotypes. The diploid mating pairs generated haploid progeny; loci were recombined with probability $\rho$ and remained in parental coupling with probability $1-\rho$. I performed recombination in every iterate of $\Delta \tau$ time units.

The mathematical formulation of the model provides no structure for defining generations and degree of overlap in survival of parents and offspring. The only intrinsic condition is that the host population doubles every $\ln (2) \approx 0.7$ nondimensional time units in the absence of parasitism and host competition. I typically used $\Delta \tau=0.2$, which implies approximately 3.5 recombination events per host doubling time. This causes much more recombination than is likely for most host populations. Thus, a recombination rate of $\rho=0.05$ probably approximates a typical amount of recombination between freely segregating loci. I shall return to this point later.

In summary, for all the results below I fixed the following parameters: $\Delta \tau=0.2, N=127, s=0.2$, a mutation rate of $10^{-5}$, an immigration rate of $10^{-1}$, an abundance of immigrants of $10^{-4}$, and a truncation level for local extinction of $10^{-8}$. I varied the following parameters: the number of specific types, $n$; parasite growth rate (includes host density), $b$; the cost of non-specific defense, $\alpha$; the cost of specific defense, $a$; the cost of virulence, $v$; the exponent determining rate of return on investment in non-specific defense, $z$, and the recombination rate, $\rho$.

The following sections focus on measurements of five variables. I assume, for each variable, that specific and non-specific components can be separately measured. This may be done by different appearance, response, or molecular indicators of genotype or physiology.

The first variable is variation in non-specific defense, which I measure simply as the standard deviation in the level of investment, $j$. The effectiveness of the defense is $j^{z}$, but measuring variation in investment provides a good indicator of polymorphism in non-specific defense.

The second variable is the frequency of specific defense. This is the probability that, for all pairs of hosts and parasites, an interaction leads to specific resistance. 
The third variable measures the cost of nonspecific defense. I calculate that as the regression of cost on non-specific defense. I define the cost expressed by an individual host as its fitness deviation relative to average fitness in the absence of parasites. For host $i j$, this observed cost is

$$
\operatorname{cost}(i j)=1-\frac{c_{i j}}{E\left(c_{i j}\right)}
$$

where $c_{i j}$ was given in eqn (2) as

$$
c_{i j}= \begin{cases}1-\alpha j, & i=0 \\ (1-a)(1-\alpha j) & i>0\end{cases}
$$

and $E$ is the expectation (average) over the frequency of host genotypes. Quantitative resistance is simply $j^{z}$. From these definitions, when average expression, $j$, is high, then $E\left(c_{i j}\right)$ is small. Thus, small changes in $j$ cause small changes in nonspecific resistance but large changes in the effective cost. Note also that an association between the cost of specific resistance, $a$, and non-specific expression, $j$, can influence the observed cost of non-specific defense. This association will be measured by the fifth variable, given below.

The fourth variable is the cost of specific resistance. I measure this effect as the regression of cost on the presence or absence of an allele that confers specific resistance. An allele confers specific resistance if it is effective against more than $1 \%$ but fewer than $99 \%$ of parasites. Presence is assigned a value of one and absence is assigned a value of zero. Cost is defined as in the previous paragraph.

The final variable is the correlation between specific and non-specific defense. I coded specific defense as a zero or one for absence or presence as defined in the previous paragraph. Non-specific resistance is $j^{z}$.

For each parameter combination, I began a run by setting the abundance of a randomly chosen host genotype to the carrying capacity. All other hosts and parasites were initially absent and were subsequently introduced by mutation or immigration. I completed initialization by iterating over 20000 non-dimensional time units, that is, $20000 / \Delta \tau$ iterations. During the following
2000 non-dimensional time units, I collected data on the five variables plus other measures. I report below on the median value of each variable over the 2000 time units for which I collected data.

\section{GENE-FOR-GENE SPECIFICITY PLUS NON-SPECIFIC DEFENSE}

I analysed this model with a factorial design over the following parameter combinations: $b=1,3,9 ; \alpha=0.2,0.4,0.8 ; z=0.4,0.8,1.6$; $a=0.05,0.1,0.2 ; v=0.05,0.1,0.2 ; \rho=0,0.005$, $0.05,0.5$. This design has $4 \cdot 3^{5}=972$ parameter combinations. I discuss the results with respect to the three questions given in the introduction to this section.

The first two questions can be taken as a pair: What parameters favor qualitative polymorphism in non-specific defense and little polymorphism for specific defense? What parameters favor polymorphism of specific defense but little variation in non-specific defense?

The answers to these two questions are consistent with the special cases discussed earlier. Nonspecific defense varies qualitatively when $z>1$ and quantitatively when $z<1$. For $z>1$, polymorphism of non-specific defense varies in accord with eqn (7). For $z<1$, quantitative variation in non-specific defense tends to increase as $z$ increases, which corresponds to a decline in the strength of stabilizing selection. Figure 3 shows variation in non-specific defense for a few parameter combinations.

Variation in specific defense is conrolled mainly by the parameters $v$ and $z$. An increase in $v$ causes a rise in specific resistance [Fig. 4(a)], as expected from the gene-for-gene models described earlier. An increase in $z$ causes a decline in specific resistance [Fig. 4(b)]. This probably occurs because a rise in non-specific defense reduces the benefit of specific resistance.

The third question is: How does the interaction between specific and non-specific defense influence the measurement of the cost of resistance? The brief answer is that selection can create very strong negative correlations between the expressions of specific and non-specific resistance. For example, an individual with specific resistance may have relatively low expression of costly nonspecific resistance. This can lead to a measured 


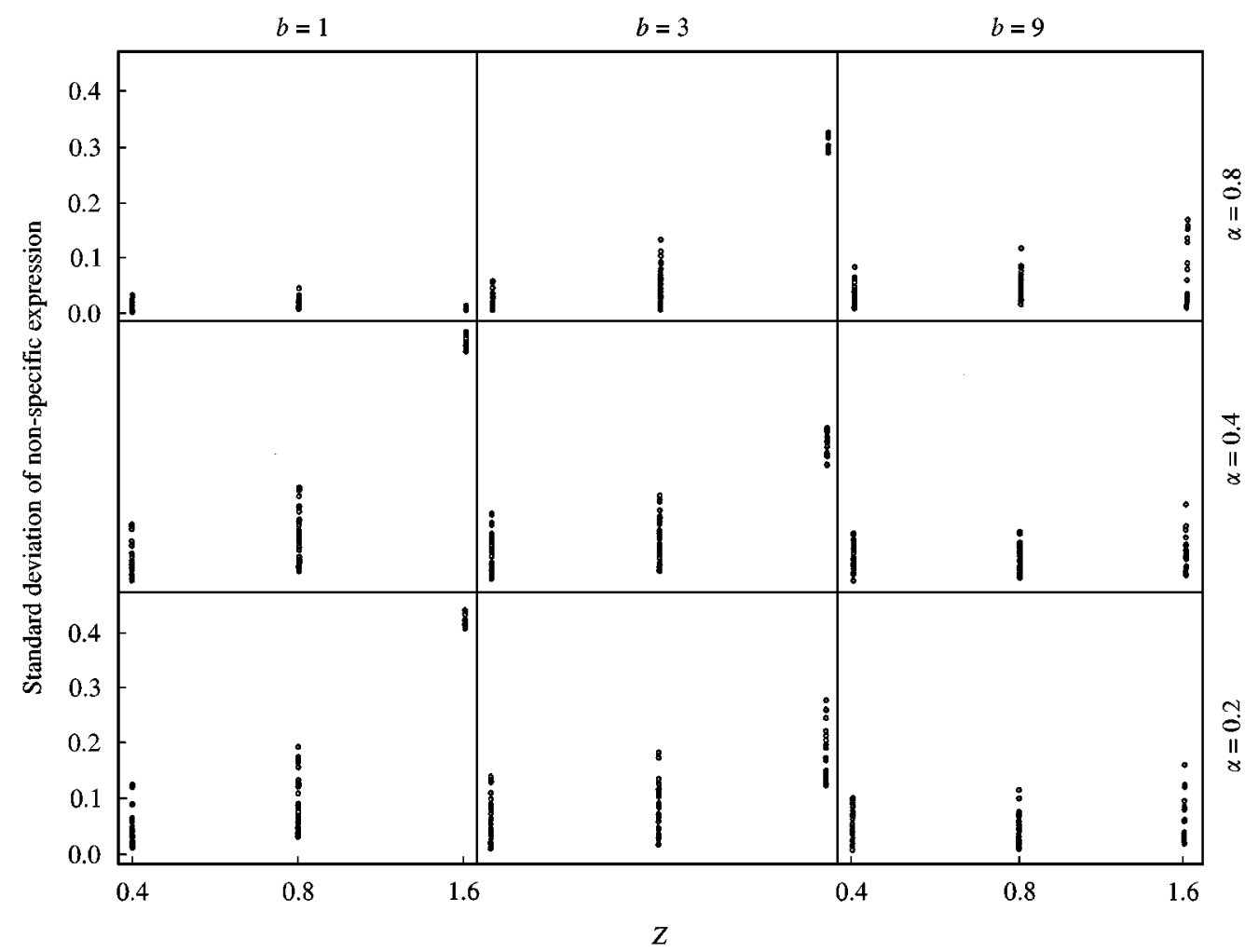

FIG. 3. Variation in non-specific defense in a model with both gene-for-gene specificity and non-specific resistance. The plots show the standard deviation in $j$, the investment in non-specific resistance. For each of the following plots, each point is the median value of the response variable over the 2000 time units of a run with a particular set of parameters. The observed values fluctuate over time, particularly as the tendency for epidemics (high $b$ ) increases. As explained in the text, six parameters were varied over 972 combinations. The parameters shown provide the best explanation for the observed variation.
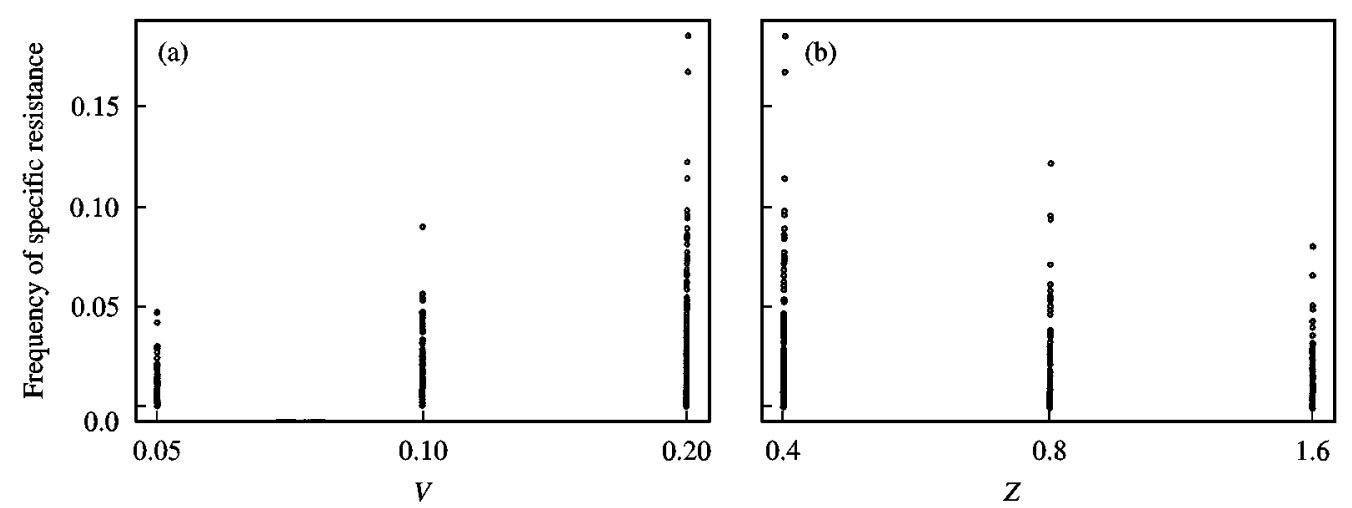

FIG. 4. Frequency of specific resistance in a model with both gene-for-gene specificity and non-specific resistance. See the legend of Fig. 3 for further details.

cost of specific resistance that is negative because, when parasites are absent, individuals with specific resistance have higher fitness than those that lack specific resistance.
Figures 5 and 6 show the measured cost of specific resistance for various parameter combinations. There are several points to be noted. Observed costs are frequently negative. The 


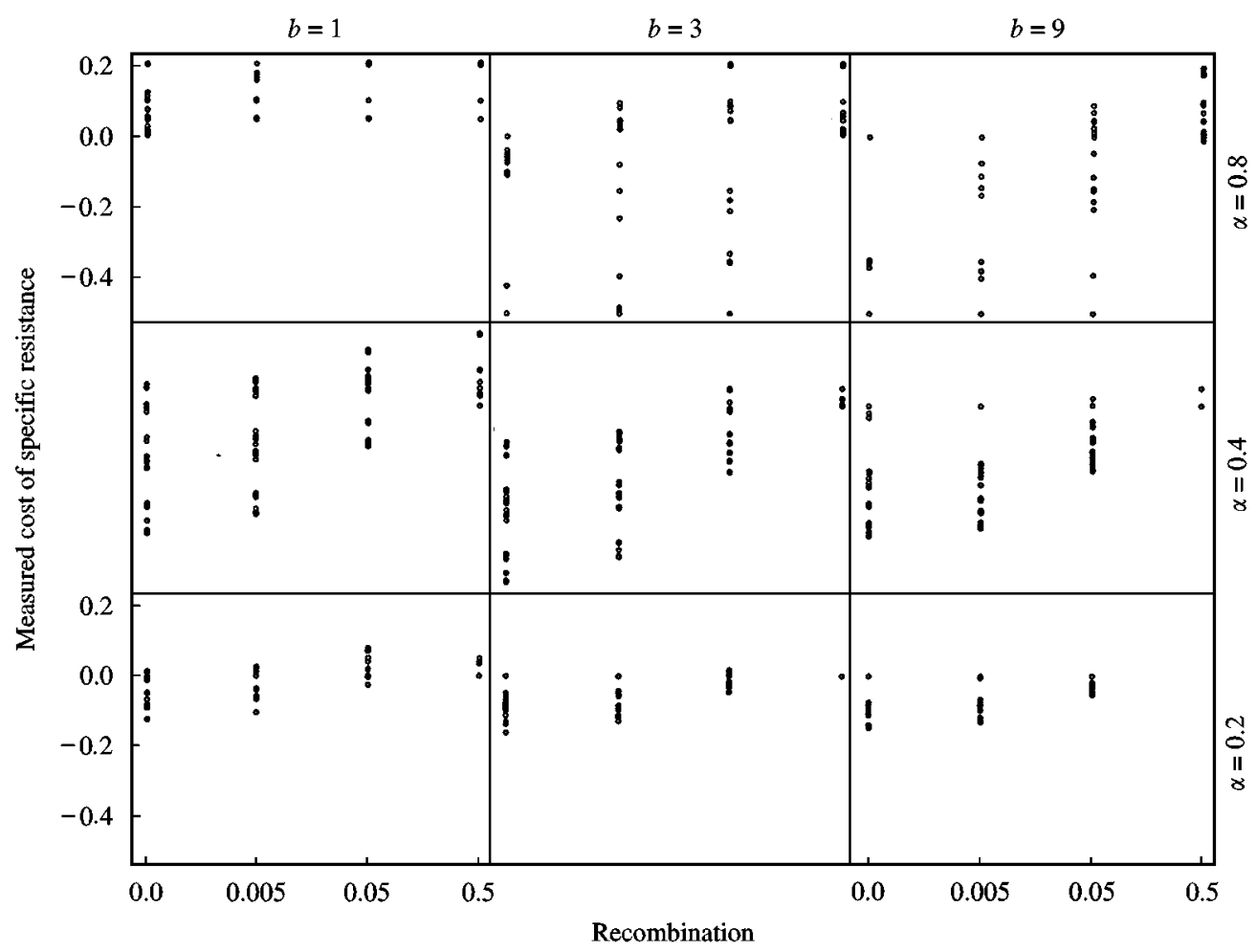

FIG. 5. Observed cost of specific resistance in a model with both gene-for-gene specificity and non-specific resistance. Values less than -0.5 were truncated to -0.5 . A value of zero was assigned when there was no variation in specific resistance. Recombination is measured by a scaled ratio of recombination to selection intensity (see text). See the legend of Fig. 3 for further details.

physiological cost of specific resistance, $a$, is not shown as an explanatory parameter because it explains little of the observed variation. An increase in $b$ is associated with greater non-equilibrium fluctuations (epidemics), more frequent negative values for observed costs, and a wider range of measured values. Higher costs of nonspecific resistance, $\alpha$, cause more frequent negative values and a wider spread. Increasing recombination moves observed costs toward positive values and reduces the range of values - the role of recombination is discussed further below.

Figure 7 shows the measured cost of nonspecific defense. The striking observation here is the very high values. These high values occur because average expression of non-specific defense can be high, causing lowered average fitness and therefore greater variation in relative fitness for variations in expression (see above for the definition of non-specific cost). Another interesting observation is that negative values only occur in the lower-left panel, for high intrinsic costs, $\alpha$, and for low recombination rates (analysis of recombination not shown). When intrinsic costs are moderate, $\alpha=0.2$, observed costs vary moderately between 0 and 0.5 .

Figure 8 shows the correlation between the presence or absence of specific defense and the level of non-specific defense (see the definition above). The correlations vary widely and are often strongly negative. Recombination explains much of the variation. When recombination is low, selection builds negative associations - individuals with strong non-specific defense are naturally favored if they do not waste resources on specific defense; those with specific defense are favored if they do not waste resources on nonspecific defense. Recombination breaks down these associations created by selection.

As mentioned above, a recombination rate of 0.5 may be much higher than free segregation. I performed complete random mating and recombination after every iterate of length $\Delta \tau=0.2$. This roughly corresponds to a generation 


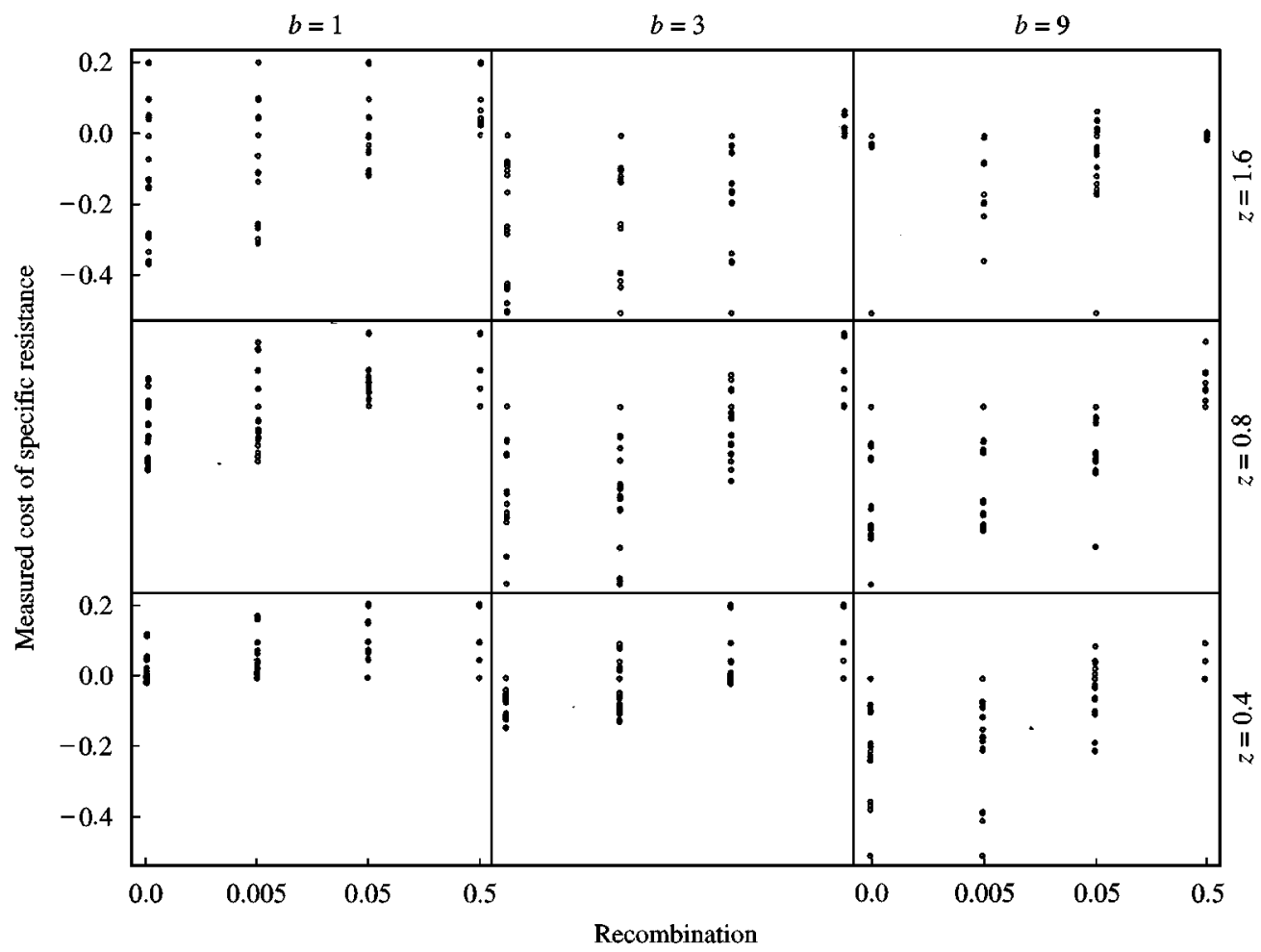

FIG. 6. Observed cost of specific resistance in a model with both gene-for-gene specificity and non-specific resistance. These plots highlight $z$ along the rows rather than $\alpha$ as in Fig. 5. Recombination is measured by a scaled ratio of recombination to selection intensity (see text). See the legend of Fig. 3 for further details.

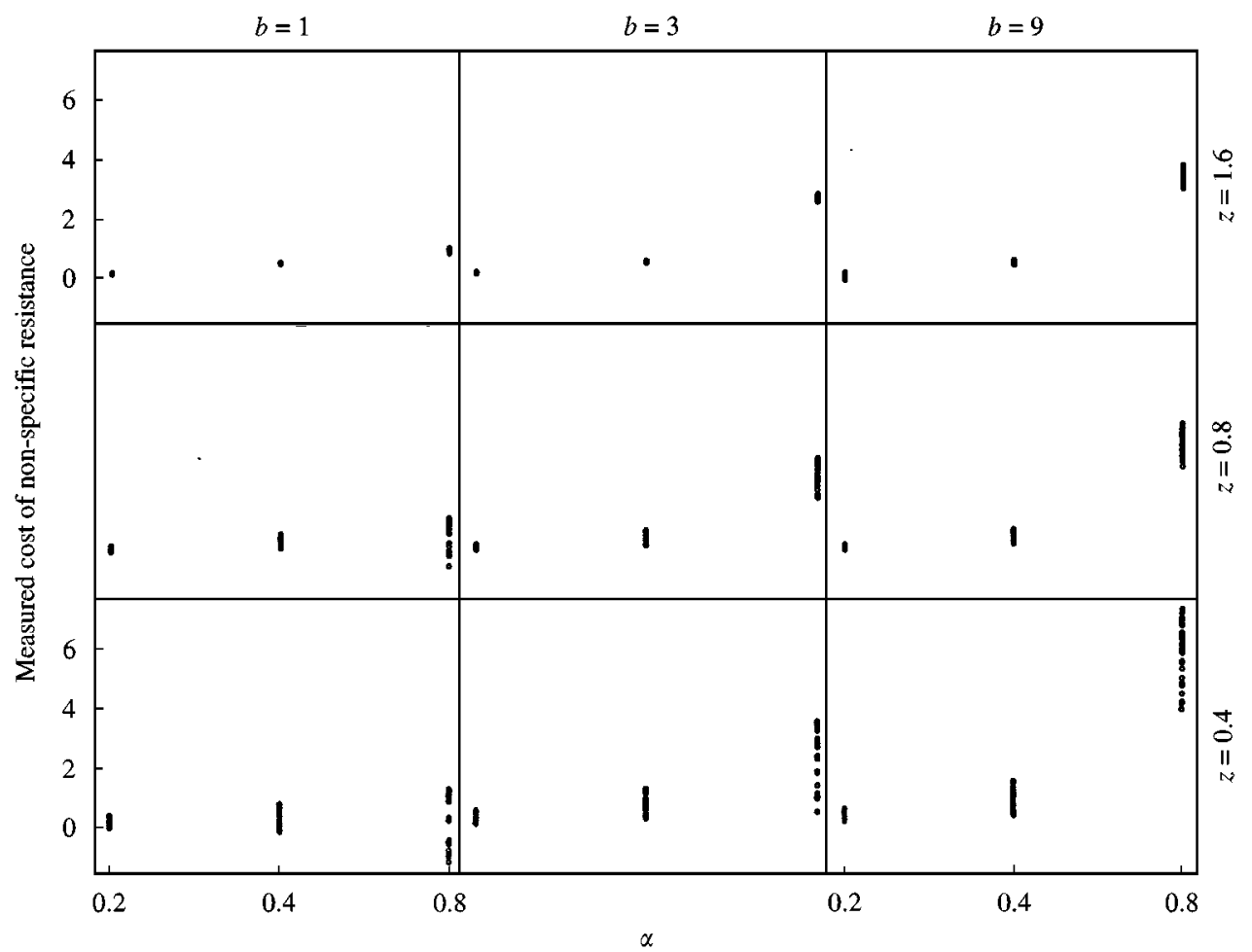

FIG. 7. Observed cost of non-specific resistance in a model with both gene-for-gene specificity and non-specific resistance. See the legend of Fig. 3 for further details. 


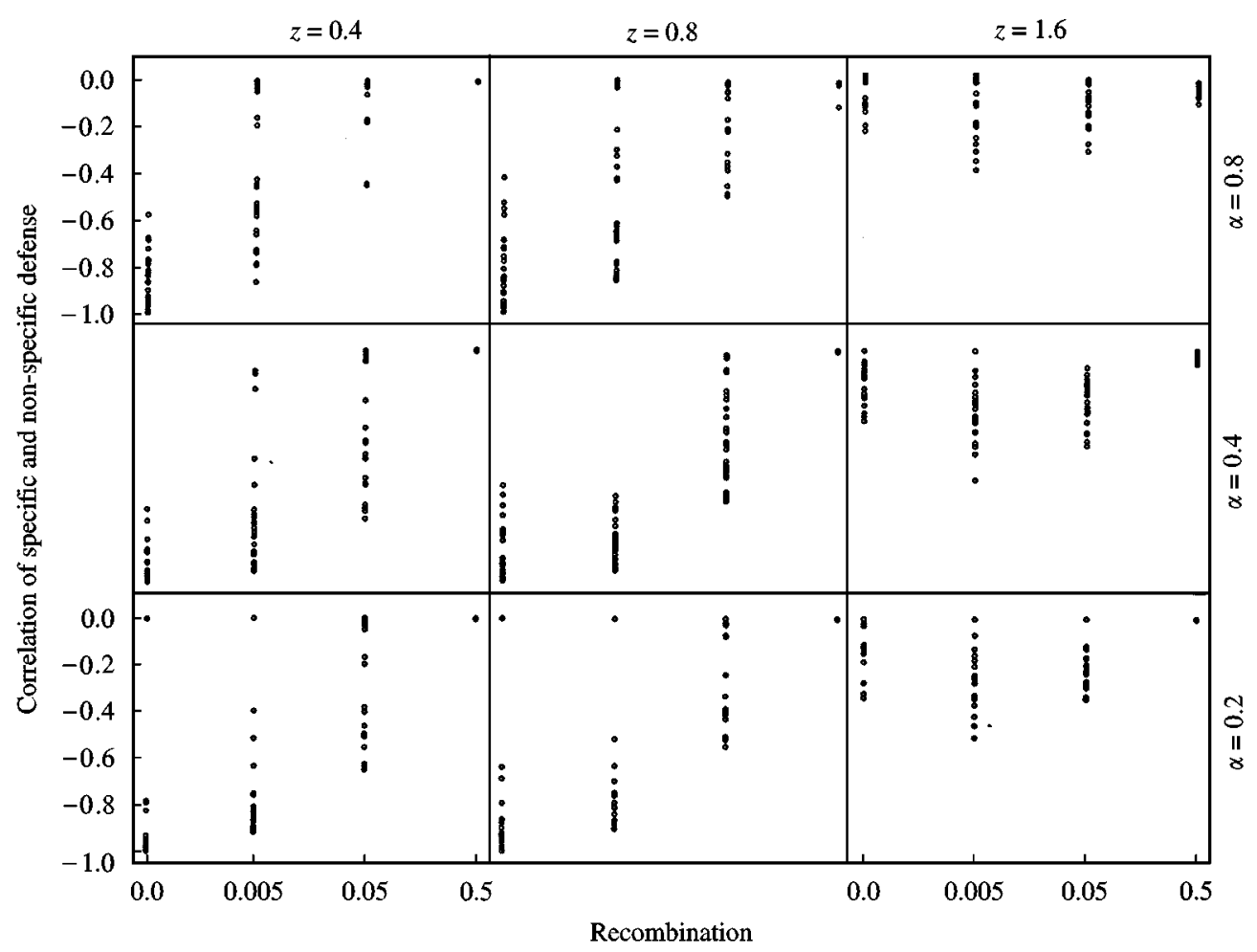

FIG. 8. Correlation of specific and non-specific resistance in a model with both gene-for-gene specificity and non-specific resistance. A value of zero was assigned when either specific or non-specific resistance lacked variation. Recombination is measured by a scaled ratio of recombination to selection intensity (see text). See the legend of Fig. 3 for further details.

time for hosts that is equivalent to the time required for the host population to increase by $e^{0.2}-1 \approx 0.22(22 \%)$ in the absence of parasites and density-dependent competition. If, by contrast, a generation of hosts corresponds to a potnetial increase of $x$, then generation time is $\ln (1+x)$, where generation time is the expected time between recombination events. For example, if a tree had a potential increase of $10^{4}$ per generation, then the expected time between mating and recombination events would be $\ln \left(1+10^{4}\right) \approx 9.2$ non-dimensional time units, or 46 times less often than imposed in my analyses. Put another way, high fecundity imposes strong selection in relation to recombination (see Discussion).

In summary, physical linkage or high fecundity of hosts per lifetime can create a strong negative association between specific and non-specific defense. Such association may lead to measurement of negative costs for specific resistance.

\section{MATCHING-ALLELE SPECIFICITY PLUS NON-SPECIFIC DEFENSE}

I analysed this model with a factorial design over the following parameter combinations: $n=3,7,15 ; b=1,3,9 ; \alpha=0.2,0.4$, $0.8 ; z=0.4,0.8,1.6 ; a=0 ; v=1 ; \rho=0,0.005$, $0.05,0.5$. This design has $4 \cdot 3^{4}=324$ parameter combinations. The combination of $a=0$ and $v=1$ excludes the host susceptibility allele (because the cost of resistance is zero) and the parasite virulence allele (because the cost of virulence is one).

This matching-allele model typically maintained maximum diversity of specific resistance. Figure 9 shows that the frequency of resistance is approximately $1 / n$ for most parameters. The cases that deviate from this equilibrium occur only when there is disruptive selection on the nonspecific defense trait, $z=1.6$, and there is no recombination, $\rho=0$. 
In the absence of non-specific defense, the matching-allele model has widely fluctuating allele frequencies (Frank, 1993b). Those fluctuations tend to increase as the parasites' capacity for increase, $b$, rises. Here the effective growth rate of the parasites is kept relatively low by an increase in non-specific defense with a rise

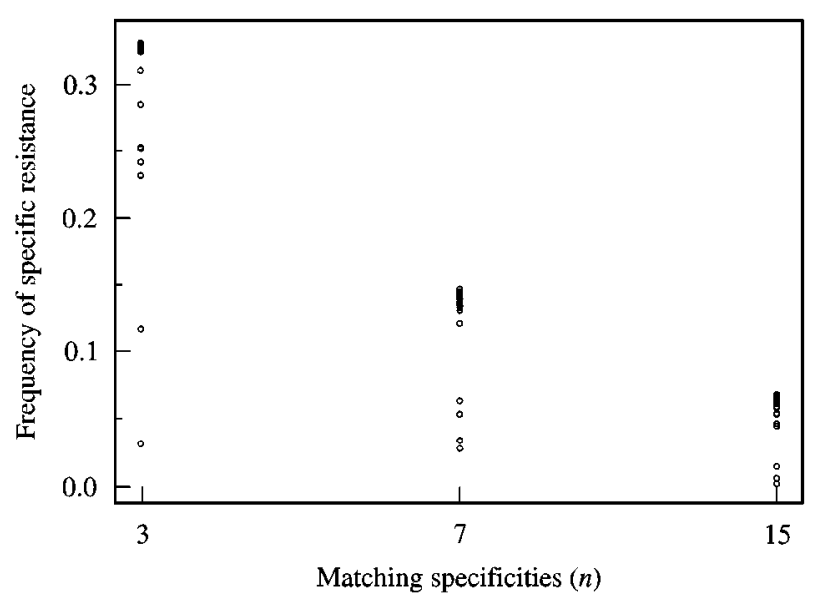

FIG. 9. Frequency of specific resistance in a model with both matching alleles and non-specific defense. in $b$ (Fig. 10). This led to an endemic parasite with nearly stable allele frequencies for matching specificities.

There was no cost for specific resistance in this model, $a=0$. Figure 11 shows the measured cost, which tends to be either close to zero or negative. In the gene-for-gene model with costly specific resistance (Figs 5 and 6), the observed costs were sometimes strongly negative. By contrast, the values here are only weakly negative. Thus, positive costs were more likely to lead to strongly negative observed values for the cost of resistance than were no costs.

What causes the contrast in measured costs between models? A cost of specific resistance favors the absence of such resistance in a host that also has strong non-specific resistance. This creates the appearance of high costs for the absence of specific resistance via the association with high levels of non-specific defense. When specific resistance has no cost, there is no pressure to associate the presence of non-specific defense with the absence of specific resistance. Consequently, the correlation between specific

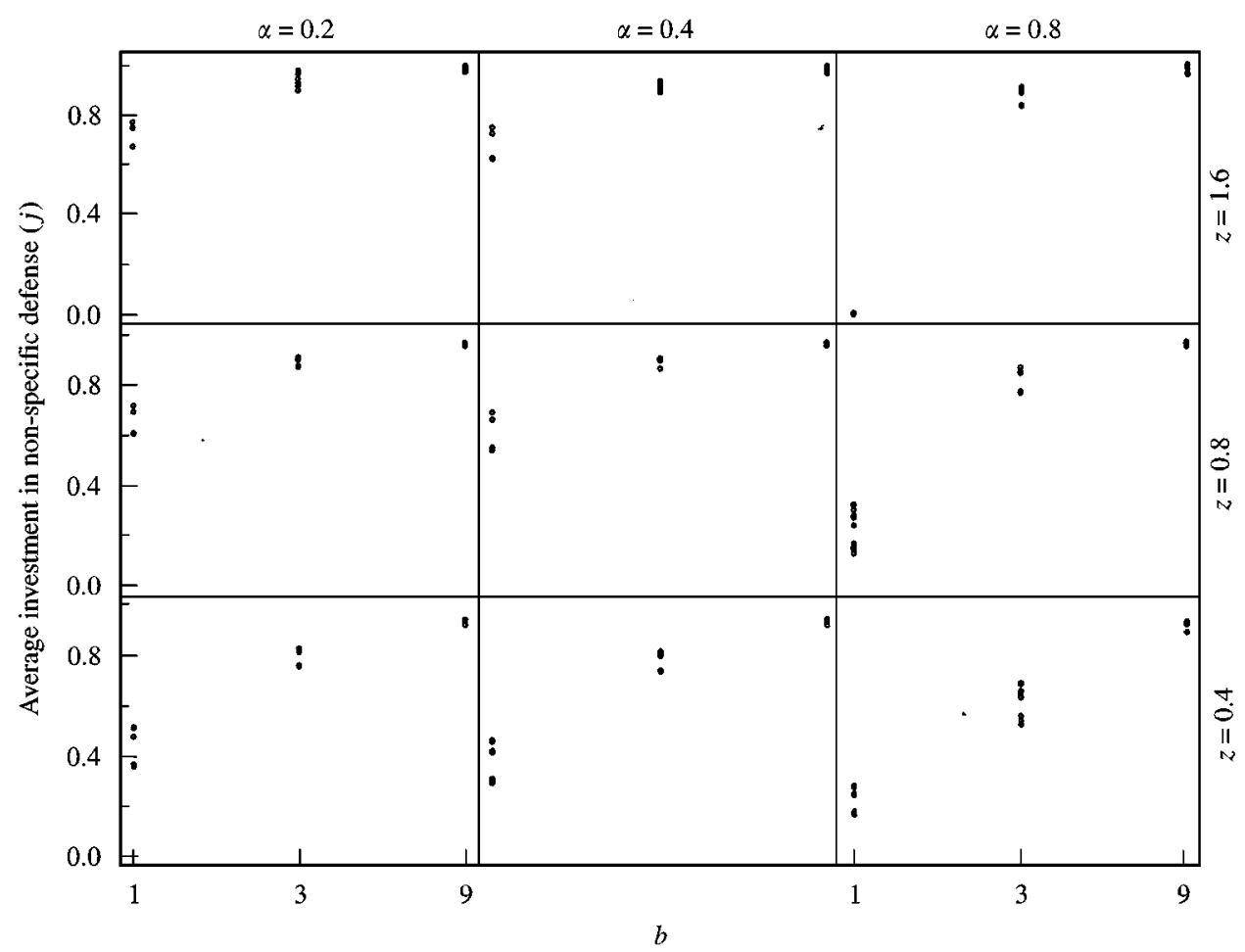

FIG. 10. Level of non-specific defense in a model with both matching alleles and non-specific defense. Average investment in non-specific defense is difficult to measure directly; one can only compare differences in defense and apparent costs between individuals. The high levels of average investment shown here explain the genetic stability near equilibrium shown in Fig. 9. 


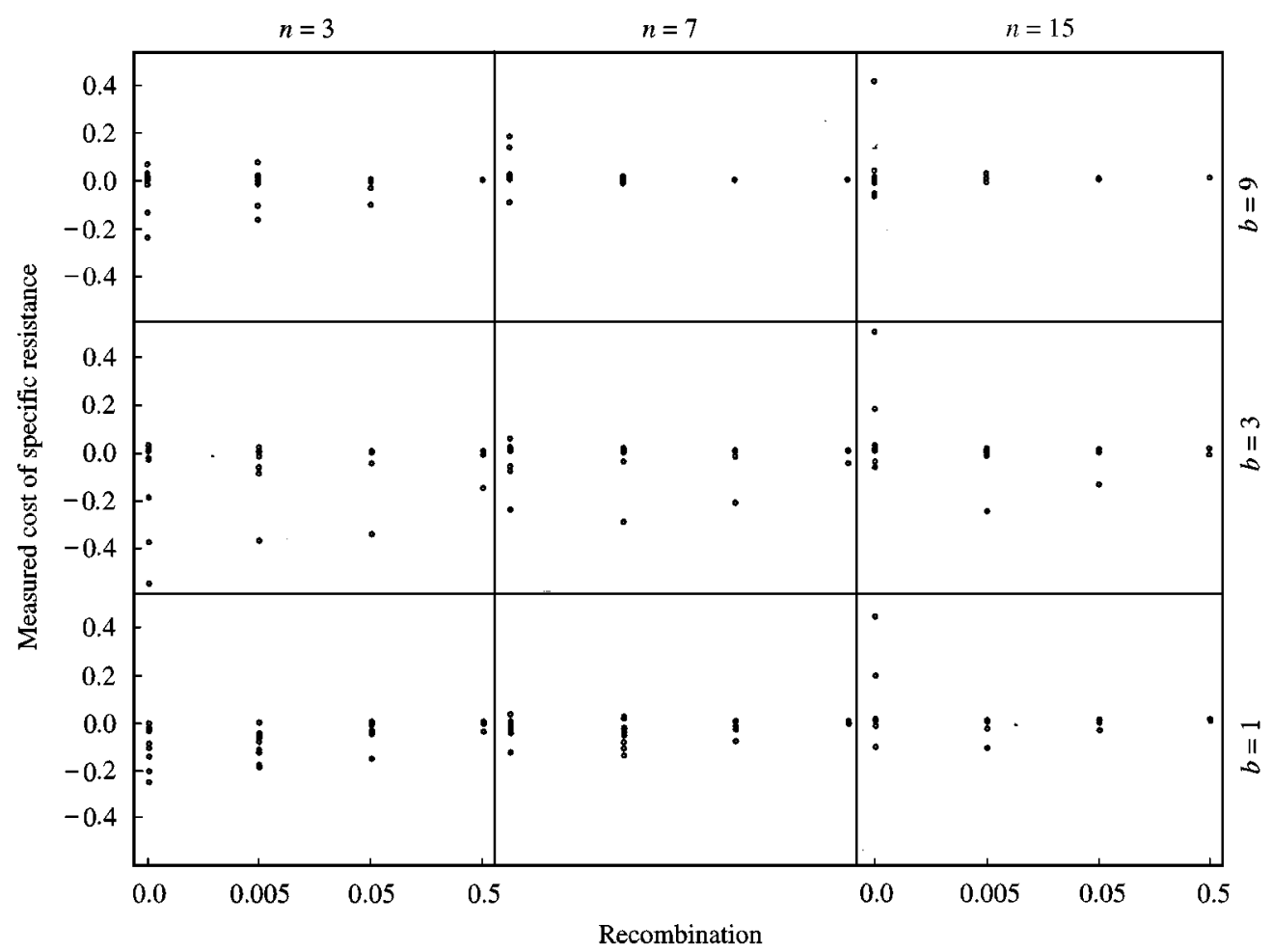

FIG. 11. Measured cost of specific resistance in a model with both matching alleles and non-specific defense. Recombination is measured by a scaled ratio of recombination to selection intensity (see text).

and non-specific defense is weak in this model (data not shown).

Finally, the measured cost of non-specific defense had a pattern similar to that shown in Fig. 7. In this case, negative values never occurred because the association between specific and non-specific defense was weak.

\section{MIXED SPECIFICITY PLUS NON-SPECIFIC DEFENSE}

The final model examines a hybrid of the genefor-gene and matching-allele assumptions for specificity plus the usual assumptions for nonspecific defense. The design repeats the prior section, but with a cost of resistance and a cost of virulence of $a=v=0.1$. The interesting contrasts from the prior models concern the frequency of specific resistance and the measured cost of specific resistance.

Figure 12 shows the frequency of specific defense, which tends to be low for all parameter combinations. There is a small amount of specific resistance maintained when the cost of non-specific defense is high and there are relatively few specificities (lower-left panel). Otherwise, the value of specific resistance is reduced by the simultaneous presence of non-specific defense to the point where the benefits do not outweigh the costs. Non-specific defense tends to be highly expressed in a pattern similar to that shown in Fig. 10.

Figure 13 shows the measured cost of specific defense. As in Fig. 5, stronger parasite pressure (high $b$ ) reduces the observed cost of resistance by causing fluctuating selection and a negative association between specific and non-specific defense. Higher costs of non-specific defense, $\alpha$, magnify these effects. Interestingly, weaker parasite pressure and high costs of non-specific defense often cause observed costs to exceed the actual costs of 0.1 (lower-right panel).

\section{Discussion}

\section{SPECIFIC VS. NON-SPECIFIC DEFENSE}

The specific defense observed by Webster $\&$ Woolhouse (1998) and the non-specific defense 


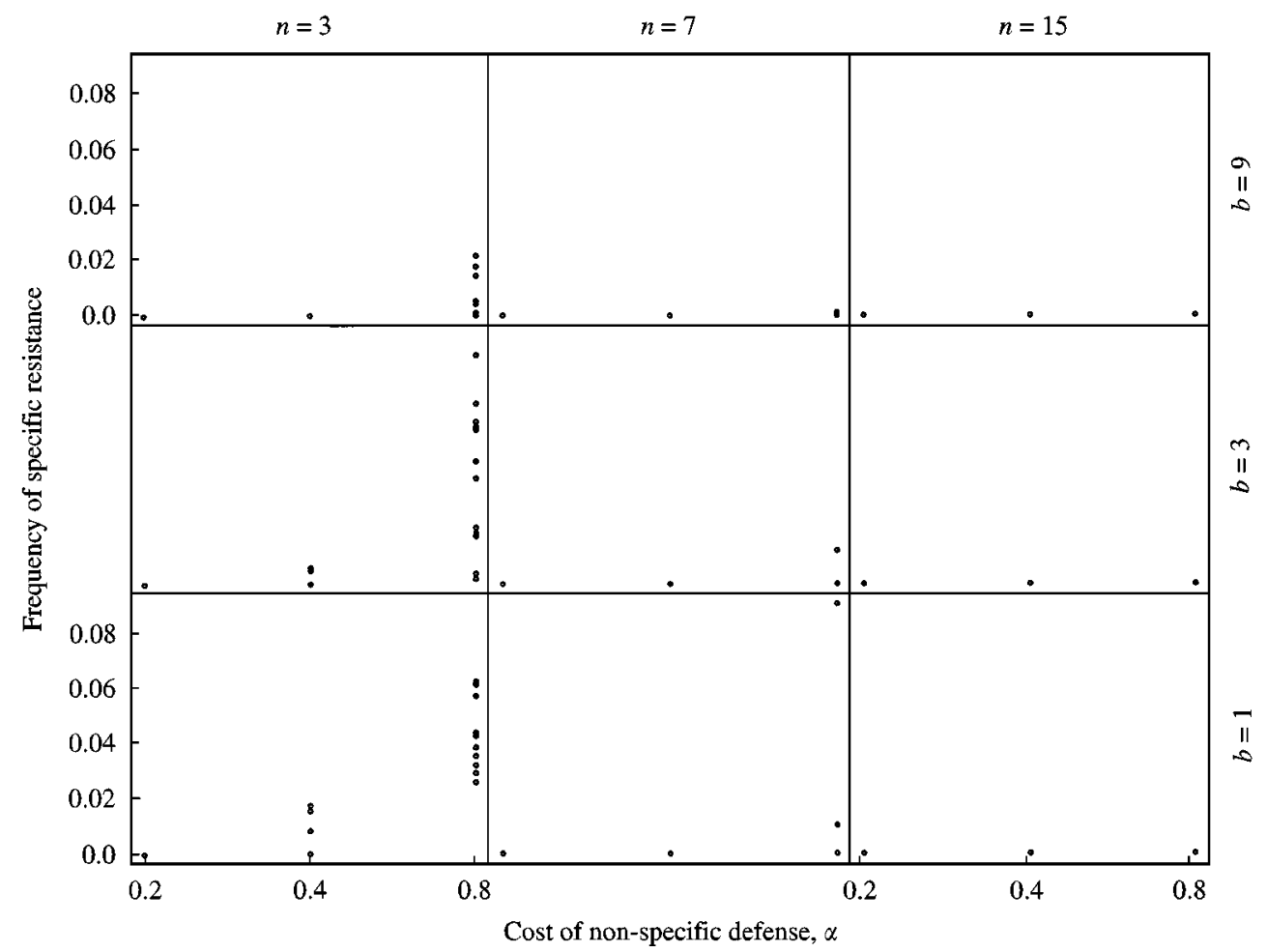

FIG. 12. Frequency of specific resistance in a model with multiple matching alleles, costly specific resistance and virulence, and non-specific defense.

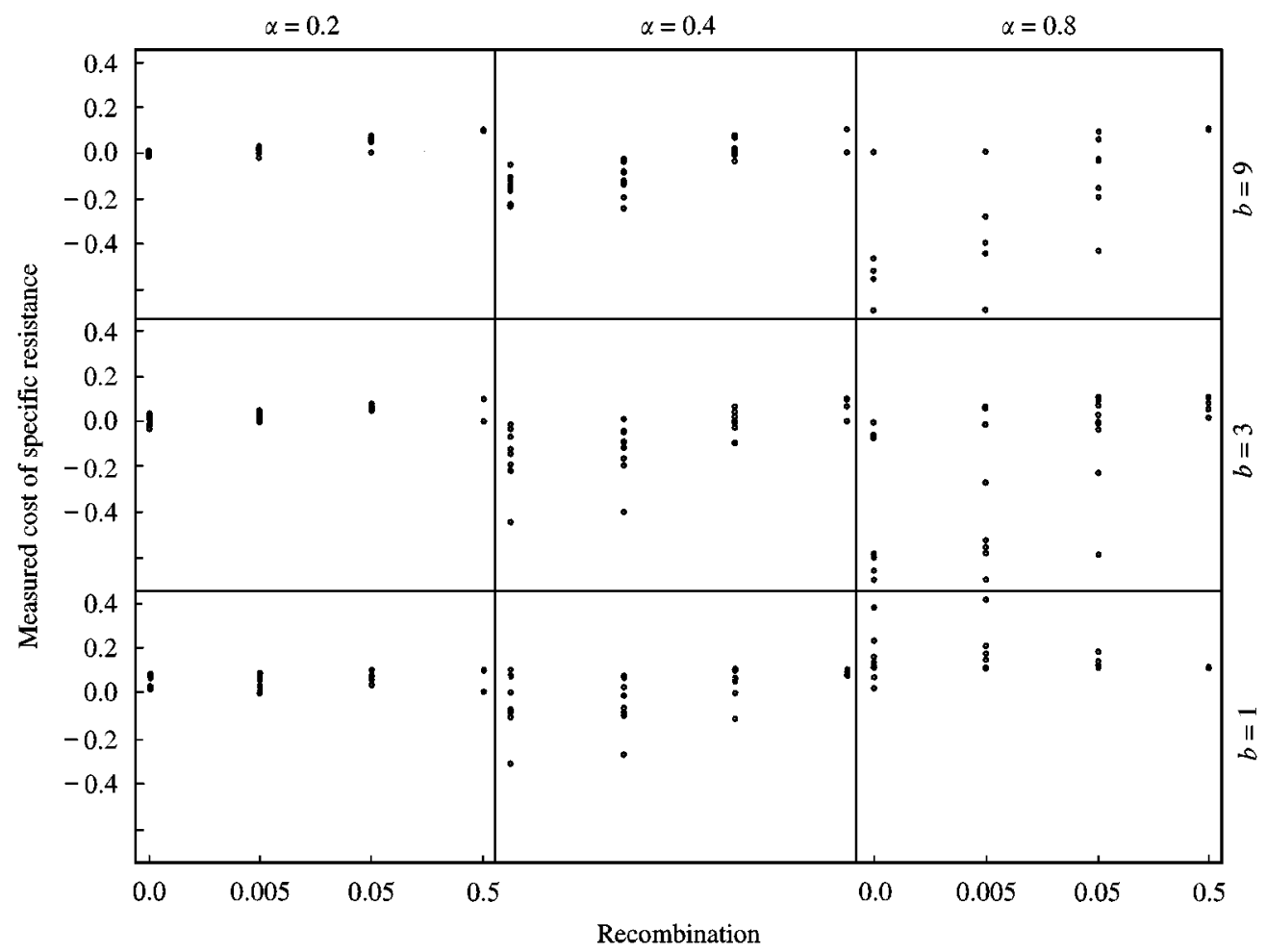

FIG. 13. Measured cost of specific resistance in a model with multiple matching alleles, costly specific resistance and virulence, and non-specific defense. Recombination is measured by a scaled ratio of recombination to selection intensity (see text). 
observed by Fellowes et al. (1998) provide an interesting contrast. Recall that Webster \& Woolhouse found all of the variation among their snail hosts to be specific against particular strains of schistosome parasites. By contrast, Fellowes et al. found variation only in non-specific defense of Drosophila melanogaster against various species of parasitoid. In addition, the non-specific defense appeared to be qualitativeeither succeeding or failing rather than graded.

There are two ways in which to consider the specificity of the Webster \& Woolhouse snails vs. the non-specific defense of the Fellowes et al., Drosophila. On the one hand, the snails may simply lack the biochemical potential to produce non-specific defense against the various schistosome strains, and D. melanogaster may lack the biochemical potential to produce specific responses to different parasitoids. On the other hand, both systems may contain the potential for specific and non-specific defense, but the genetic and epidemiological parameters may favor specificity in the case of snails and variable non-specific defense in the case of Drosophila.

One cannot choose between the biochemical potential and the different parameter alternatives based solely on theoretical analysis. But the widespread distribution of both specific and nonspecific defense suggests that biochemical potential is often not the limiting factor. Thus, it is useful to consider what parameters would favor one type of defense over the other.

What parameters favor the observation of specific defense, as in the case of the snails? When benefits of non-specific defense increase in a diminishing way with expression, $z<1$, then variation tends to be limited to small, quantitative increments (Fig. 3). If specific variation is also present, these small levels of quantitative variation in non-specific defense may be overlooked during selection experiments that emphasize large, qualitative effects. Variation in specific defense is maintained unless: parasites can produce a universal host-range allele with very low cost of virulence $(v \rightarrow 0)$; or there are several alternative matching specificities and the hosts pays a significant cost of resistance, $a$. These conditions can be roughly summarized as $z<1, v>0$, and $a \ll 1 / n$, where $n$ is the number of matching specificities.
What parameters favor the observation of rare, qualitative, non-specific defense, as in the case of D. melanogaster and its parasitoids? When benefits of non-specific defense increase in an accelerating way with expression, $z>1$, then variation in non-specific defense is qualitative and influenced in frequency by the conditions in eqn (7). In particular, non-specific defense declines as the cost, $\alpha$, rises and as the potential rate of increase of the parasite, $b / s$, declines, that is, costly defense and endemic parasites with a relatively low rate of potential increase. These conditions appear to match the situation for the $D$. melanogaster-parasitoid interaction: Fellowes et al. (1998) found that defense was indeed costly, and parasitoids have relatively low rates of increase compared to other kinds of parasites. The conditions for limited variation in specific defense are, inverting from the previous paragraph, $v \rightarrow 0$ or $a$ not much less than $1 / n$.

I suggested above that biochemical potential probably does not, by itself, exclude either specific or non-specific defense. But the biochemistry of the host-parasite interaction certainly must influence some of the key parameters, such as the number of matching specificities and the costs of resistance and virulence. Thus, full knowledge of population genetics and epidemiology depends on biochemical and mechanistic knowledge; likewise, the biochemistry and mechanisms of host-parasite interaction only have meaning when embedded within the dynamics of population genetics and epidemiology (Frank, 1994). This mutual dependence between the physical mechanisms of interaction and the population biology is further illustrated by the costs of resistance.

\section{MEASURING THE COSTS OF RESISTANCE}

The theory emphasizes costs of resistance in determining evolutionary outcome. Much effort at measurement and discussion of significance have been attached to this subject (Simms, 1992). The analyses here make clear that even perfect measurement of natural populations can be very misleading about the intrinsic (or mechanistic) level of costs.

The key difficulty is that selection powerfully builds negative associations between specific and 
non-specific defense or, more generally, between the many different components of the defensive arsenal. Individuals that are particularly well defended by any single defensive component do best when they have strongly reduced expression of other costly components of defense. In the models presented here, specific defense with an intrinsic (mechanistic) cost often appeared to have a negative cost - that is, specific defense was associated with higher fitness in the absence of parasites (Figs 5, 6 and 13). Negative associations between specific and non-specific defense are illustrated in Fig. 8.

What conclusions can be drawn? Variable intrinsic costs provide comparative predictions about changing pattern; comparative patterns observed in natural populations suggest hypotheses about variable intrinsic costs. But costs are difficult to measure by population analyses; we ultimately depend on future physiological and biochemical studies. These future studies, with estimated costs, must then be fed back into population-level analyses such as those presented here.

What should be done before mechanistic estimates for costs are available? Probably the most fruitful line of research will be comparisons among populations and among systems. I set up the Drosophila vs. snail comparison to show a strong contrast between very different systems. Studies of different snails and different Drosophila will begin to fill in a richer comparative dataset. The population outcomes will then suggest hypotheses about intrinsic costs, which can ultimately be tested by physiological and biochemical studies.

\section{ASSOCIATIONS BETWEEN SPECIFIC AND NON-SPECIFIC DEFENSE}

I have emphasized that selection creates a negative association between costly components of a defensive system. An individual, successful in one component of defense, gains by reducing costly expression of other forms of resistance.

The state of the population depends on the balance between the rate at which selection creates associations and the rate at which recombination breaks up those associations. Recombination is usually expressed as one minus the probability that a pair of loci in a parent will be transmitted together to an offspring - the rate at which loci are separated per generation. Free recombination corresponds to independent assortment of loci, that is, a recombination probability of 0.5 per generation.

The model I used scaled time according to the hosts' intrinsic rate of increase, $r$, rather than to generation time. This effectively scales time and the strength of selection to the hosts' potential doubling rate rather than to the tempo of mating, birth, and death. As explained in the text, each time step at which I applied recombination corresponded to the time required for the host population to increase by approximately $22 \%$ in the absence of competition and parasitism. If a host population could increase by only $22 \%$ per generation, then the recombination rate applied corresponds to the per-generation recombination rate. By contrast, if a population had the potential to increase by a larger factor per generation, then the strength of selection rises and the effective recombination-selection ratio declines.

The recombination parameter shown in the figures is the recombination-selection ratio (Figs 5, 6, 8, 11 and 13). For example, a value of 0.05 corresponds roughly to free recombination (0.5) in each generation for a population that could potentially increase by a factor of 6.4 in each generation (see calculations in the section Gene-for-gene specificity plus non-specific defense). The model is not designed for these numbers to be exact, but one can extract the approximate trends for the recombination-selection scaling. The point is that strong selection can create negative associations even when recombination is high.

In this model, separate loci encode the two independently expressed components of specific and non-specific defense. Associations between levels of expression can only arise passively by statistical processes of selection and recombination. Clearly, hosts can gain an advantage by coupling different components into a common regulatory network. For example, costly nonspecific expression may follow only after a trigger by recognition of invasion. The model presented here shows how the early stages of association among components may have arisen by statistical coupling of independent components. This sets the stage for modifiers of regulatory control 
that can enforce coupling by controlled responses to particular stimuli. It may be that inducible defense and other complex defensive cascades arose in this way.

This research is supported by National Science Foundation grant DEB-9627259.

\section{REFERENCES}

Boots, M. \& HARAGUCHI, Y. (1999). The evolution of costly resistance in host-parasite systems. Am. Nat. 153, 359-370. Burdon, J. J. (1987). Diseases and Plant Population Biology. Cambridge: Cambridge University Press.

Fellowes, M. D. E., KraAiJeveld, A. R. \& Godfray, H. C. J. (1998). Trade-off associated with selection for increased ability to resist parasitoid attack in Drosophila melanogaster. Proc. Roy Soc. Lond. B 265, 1553-1558.

Fellowes, M. D. E., KraAijeveld, A. R. \& Godfray, H. C. J. (1999). Cross-resistance following selection for increased defense against parasitoids in Drosophila melanogaster. Evolution 53, 1302-1305.

FLOR, H. H. (1971). Current status of the gene-for-gene concept. Ann. Rev. Phytophath. 9, 275-296.

FRANK, S. A. (1992). Models of plant-pathogen coevolution. Tr. Genetics 8, 213-219.

FRANK, S. A. (1993a). Coevolutionary genetics of plants and pathogens. Evol. Ecol. 7, 45-75.

FRANK, S. A. (1993b). Specificity versus detectable polymorphism in host-parasite genetics. Proc. Roy. Soc. Lond. B 254, 191-197.

FRANK, S. A. (1994). Recognition and polymorphism in host-parasite genetics. Phil. Trans. Roy. Soc. Lond. B 346, 283-293.

FrANK, S. A. (1997). Spatial processes in host-parasite genetics. In: Metapopulation Biology: Ecology, Genetics and Evolution (Hanski, I. \& Gilpin, M., eds), pp. 325-352. New York: Academic Press.
Gabriel, D. W. \& Rolfe, B. G. (1990). Working models of specific recognition in plant-microbe interactions. Ann. Rev. Phytopath. 28, 365-391.

Harvell, C. D. (1990). The ecology and evolution of inducible defense. Q. Rev. Biol. 65, 323-340.

KARBAN, R. \& BALDWIN, I. T. (1997). Induced Responses to Herbivory. Chicago: University of Chicago Press.

Kearney, B., Ronald, P. C., DAhlbeck, D. \& StasKAWICZ, B. J. (1988). Molecular basis for evasion of plant host defense in bacterial spot disease of pepper. Nature 332, 541-543.

KraAijeveld, A. R., van Alphen, J. J. M. \& Godfray, H. C. J. (1998). The coevolution of host resistance and parasitoid virulence. Parasitology 116, S29-S45.

LEONARD, K. J. \& CzOCHOR, R. J. (1980). Theory of genetic interactions among populations of plants and their pathogens. Ann. Rev. Phytopath. 18, 237-258.

May, R. M. (1974). Stability and Complexity in Model Ecostystems, 2nd Edn. Princeton: Princeton University Press.

Murray, J. D. (1989). Mathematical Biology. New York: Springer-Verlag.

SAlT, G. (1970). The Cellular Defence Reactions of Insects. Cambridge: Cambridge University Press.

Sasaki, A. \& Godfray, H. C. J. (1999). A model for the coevolution of resistance and virulence in coupled hostparasitoid interactions. Proc. Roy Soc. Lond. B. 266, 455-463.

SEGEL, L. A. (1972). Simplification and scaling. SIAM Rev. 14, 547-571.

Simms, E. L. (1992). Costs of plant resistance to herbivory. In: Ecology and Evolution of Plant Resistance (Fritz, R. S. \& Simms, E. L., eds), pp. 392-425. Chicago: University of Chicago Press.

Tollrian, R. \& Harvell, C. D. (1999). The Ecology and Evolution of Inducible Defenses. Princeton: Princeton University Press.

VAnderplank, J. E. (1963). Plant Diseases: Epidemics and Control. New York: Academic Press.

VAnderplank, J. E. (1984). Disease Resistance in Plants, 2nd Edn. New York: Academic Press.

Webster, J. P. \& Woolhouse, M. E. J. (1998). Selection and strain specificity of compatibility between snail intermediate hosts and thier parasitic schistosomes. Evolution 52, 1627-1634. 